\title{
Configuration of Antiphase Domains in One-Dimensional Long Period Superstructure Alloys
}

\author{
By Tetsuo Eguchi*, Yoshitsugu Tomokiyo** and Noriyuki Kuwano*
}

\begin{abstract}
Lattice images of some alloys with one-dimensional long period superstructures based on $f c c$ lattices were observed by means of a high resolution electron microscope JEM-1000. The observed lattice images as compared with their corresponding computer simulations revealed the details of antiphase domain structures in the alloys. In particular the sites of minority atoms usually appear with contrast in the images. The alloys with nonintegral mean values of domain sizes actually consist of two kinds of domains whose sizes are either one of the two adjacent integers. The way of mixture of two kinds of domains was examined in the images, and a tentative model to describe the sequence and the distribution of domain sizes was presented. Some remarks were added on the roles of jogs of antiphase boundaries in the image formation.
\end{abstract}

(Received November 30, 1982)

Keyzerds: lattice image, one-dimensional long period superstructure, antiphase domain boundary

\section{Introduction}

A group of alloys, which are typically represented by the $\mathrm{Cu}-\mathrm{Au}$ system, form superstructures based on an $f c c$ lattice. These alloys sometimes exhibit so-called one-dimensional long period superstructures, with antiphase boundaries (APB) inserted periodically. The average value of the distances between two successive APB, or the half period of the structure, can be obtained by diffraction experiments. The half period, denoted as $\bar{M}$ herafter, depends upon the kind of alloy and its composition ${ }^{(1)}$. Measured values of $\bar{M}$ usually turn out not to be integers or halfintegers in terms of the unit of basic $f c c$ lattice parameter. The antiphase structures of nonintegral or non-half-integral $\bar{M}$ values have long been discussed intensively. For example, the $\alpha_{2}$ phase of the $\mathrm{Cu}-\mathrm{Al}$ system forms a one-dimensional superstructure called $\mathrm{D}_{22}$ type, and the measured value of $\bar{M}$, as determined by X-ray or electron diffraction, varies continuously from 4.0 for 22.0 at $\% \mathrm{Al}$

* Department of Materials Science and Technology, Graduate School of Engineering Sciences, Kyushu University, Kasuga-shi 816, Japan.

** Research Laboratory of High Voltage Electron Microscope, Kyushu University, Fukuoka-shi 812, Japan. up to 4.7 for 24.0 at $\% \mathrm{Al}^{(2)(3)}$. The observed change in $\bar{M}$ is in good agreement with the electron theoretical prediction by Sato and Toth $^{(4)}$. On the other hand, for a crystallographic interpretation of $\mathrm{CuAuII}$ alloy $(\mathrm{CuAu}$ with a long period superstructure) Ogawa et $a{ }^{(5)}$ observed the alloy under an electron microscope, and found fringes which seemed to correspond to APB. From the histogram for distribution of distances between these fringes, they concluded that the alloy with $\bar{M}=5.4$ actually consists of domains of local size of $M=5$ or 6 . Pashley et al. ${ }^{(6)}$, however, reported that they did not find anyone of such results in a similar experiment. One of the reasons for the discrepancy may be attributed to the insufficient resolving power of electron microscopes used in those days, and it seems worthwhile to re-examine the problem of antiphase structures in the long period superstructural alloys with the use of present day microscopes, whose resolving power has recently been improved amazingly to the sizes of atoms.

The purpose of the present investigation is two-fold; one is to demonstrate and analyze the lattice images of superstructural alloys with high resolution, which are photographed by the microscope JEM-1000 at the Research Laboratory of High Voltage Electron Microscope of Kyushu University, and the other to find any physical implication for the descrip- 
tion of antiphase domain structures in the group of one-dimensional long period superstructural alloys of various kinds.

In the present paper a computer simulation for the multibeam lattice images of superstructural $\mathrm{Ag}-\mathrm{Mg}$ alloys is described in order to clarify the correspondence between the actual atomic structure and its lattice image. The observed lattice images of $\alpha^{\prime} \mathrm{Ag}-\mathrm{Mg}, \alpha^{\prime \prime}$ $\mathrm{Cu}-\mathrm{Pd}$ and $\alpha_{2} \mathrm{Cu}-\mathrm{Al}$ are analyzed to obtain informations as to their antiphase domain structure, and to examine the way of arrangement of domains.

\section{Experimental Method}

The alloy specimens were rolled, chemically thinned and punched to discs $0.1 \mathrm{~mm}$ in thickness and $3 \mathrm{~mm}$ in diameter. These specimens were quenched from an elevated temperature, and were annealed at $630 \mathrm{~K}$ for one to three months, in order to grow up their long period superstructures. For electron microscopic observations the specimens were jet-polished into thin films in an electrolytic solution, for example, of perchloric acid plus methanol or nital in the case of $\mathrm{Ag}-\mathrm{Mg}$ or $\mathrm{Cu}-\mathrm{Al}$, correspondingly.

The samples thus prepared were observed under a high voltage electron microscope JEM-1000 at the Research Laboratory of High Voltage Electron Microscope, Kyushu University, equipped with a goniometer of sideentry type and a hair-pin filament. The accelerating voltage of the electron microscope was either 500,750 or $1000 \mathrm{kV}$.

\section{Computer Simulation}

For computer simulation of the lattice images for $\alpha^{\prime} \mathrm{Ag}-\mathrm{Mg}$ system photographed under the many beam condition an ordinary multislice method ${ }^{(7)(8)}$ was used. The scattering factors for $\mathrm{Ag}$ and $\mathrm{Mg}$ atoms were obtained from the formulas and parameters given by Doyle and Turner ${ }^{(9)}$, and the projected potential $\phi(x)$ at a point $\boldsymbol{x}$ was obtained for a slice of the alloy crystal of thickness $\Delta z$.

The scattering amplitude of electron wave from the slice is given by the expression:

$$
Q(\boldsymbol{h})=\mathscr{F} \exp \left\{i \sigma \phi(\boldsymbol{x})\left(1+i K_{a}\right)\right\},
$$

where $\mathscr{F}$ denotes an operator of Fourier transformation, $\sigma$ the interaction parameter with relativistic correction, and $K_{a}$ the absorption parameter. In the present calculation the thickness of the slice $\Delta z$ was taken as $a$ or $2 a$, where $a$ is the lattice parameter, and the absorption parameter as $K_{a}=0.05$. If we denote the scattering amplitude of the electron wave at the bottom of the $m$-th slice as $U_{m}(h)$, then the one of the $(m+1)$-th slice is given by

$$
U_{m+1}(\boldsymbol{h})=\left\{U_{m}(\boldsymbol{h}) \cdot P(\boldsymbol{h})\right\} \otimes Q(\boldsymbol{h}),
$$

where $\otimes$ is a convolution operator, whereas the dot a simple product. $P(\boldsymbol{h})$ is called a propagation function, and is given approximately by the following expression:

$$
P(\boldsymbol{h})=\exp \left\{-2 \pi i \Delta z\left(\lambda \boldsymbol{h}^{2} / 2-\boldsymbol{\alpha} \cdot \boldsymbol{h}\right)\right\},
$$

where $\lambda$ is the wavelength of electron beam, and $\alpha$ a unit vector representing inclination of the crystal. If we define the scattering amplitude at the bottom of the crystal by $U_{M}(\boldsymbol{h})$, the amount of defocus by $f_{\Delta}$, and the parameter for spherical abberation of the objective lense by $C_{s}$, then the image intensity $I(x)$ for the lattice image is given as ${ }^{(10)(11)}$

$$
\begin{aligned}
I(\boldsymbol{x})= & \sum_{h} \sum_{h^{\prime}} U_{M}(\boldsymbol{h}) U_{M}^{*}\left(\boldsymbol{h}^{\prime}\right) A(\boldsymbol{h}) A\left(\boldsymbol{h}^{\prime}\right) \\
& \times \exp \left[-(\pi \lambda \Delta)^{2}\left(H^{2}-H^{\prime 2}\right)^{2} / 2-2\left(\pi q_{0}\right)^{2}\right. \\
& \times\left\{C_{s} \lambda^{3}\left(H^{2} \boldsymbol{H}-H^{\prime 2} \boldsymbol{H}^{\prime}\right)-f_{\Delta} \lambda\left(\boldsymbol{H}-\boldsymbol{H}^{\prime}\right)\right\}^{2} \\
& \left.+i\left\{X(\boldsymbol{H})-X\left(\boldsymbol{H}^{\prime}\right)-2 \pi\left(\boldsymbol{H}-\boldsymbol{H}^{\prime}\right) \cdot \boldsymbol{x}\right\}\right],
\end{aligned}
$$

where $\boldsymbol{H}=\boldsymbol{h}-\boldsymbol{H}_{0}$ and $\boldsymbol{H}^{\prime}=\boldsymbol{h}^{\prime}-\boldsymbol{H}_{0}$, with $\boldsymbol{H}_{0}$ the optical axis of the microscope, $A(\boldsymbol{h})$ the aperture function which is either 1 or 0 , depending whether $\boldsymbol{h}$ is inside or outside the aperture. $\exp [i X(\boldsymbol{H})]$ is the transfer function for the objective lense, where the phase $X(H)$ is given by ${ }^{(12)}$

$$
X(\boldsymbol{H})=\pi \lambda\left(f_{\Delta} H^{2}-C_{s} \lambda^{2} H^{4} / 2\right)
$$

In the expression (4) the uncertainties of focus and convergency of the incident beam are assumed given by Gaussian functions with standard deviations $\Delta$ and $q_{0}$, respectively.

When we calculate the intensity distribution in lattice images by the use of multislice method, it is important how many diffracted waves are taken into account. In the present simulation 
we considered all the diffracted waves either inside a circle, $h^{2}+k^{2} \leqq 100$, or in a square, $-10 \leqq h \& k \leqq+10$. In the case of $\mathrm{Au} 30$ to 40 $\mathrm{nm}$ in thickness the above condition has been confirmed by the present authors to be a good approximation.

\section{Multibeam Lattice Images of Ordered Alloys}

Figure 1 shows an example of the computed intensities of transmitted and diffracted beams through $\mathrm{Ag}-\mathrm{Mg}$ as functions of the sample thickness. The sequence of antiphase domains we assumed in this case was $2 \overline{2} 2 \overline{1} 2 \overline{2} 2 \overline{1} \cdots$; namely, seven $f c c$ cells compose a unit structure with the first two in-phase, the second two anti-phase, the next two in-phase, and then the last one anti-phase. In Fig. 1 we see that the intensity of transmitted wave, or $h k l=000$, decreases with increasing thickness $T$ of the sample, whereas the one of a fundamental reflection, or $h k l=200$, increases oscillatorily, reaches the maximum at about $T=20 \mathrm{~nm}$, and then decreases. On the other hand, the intensities of superlattice reflections, such as $h k l=100$ or $2 / 7,1,0$, begin to increase gradually from $T=15 \mathrm{~nm}$, and become larger than the one for

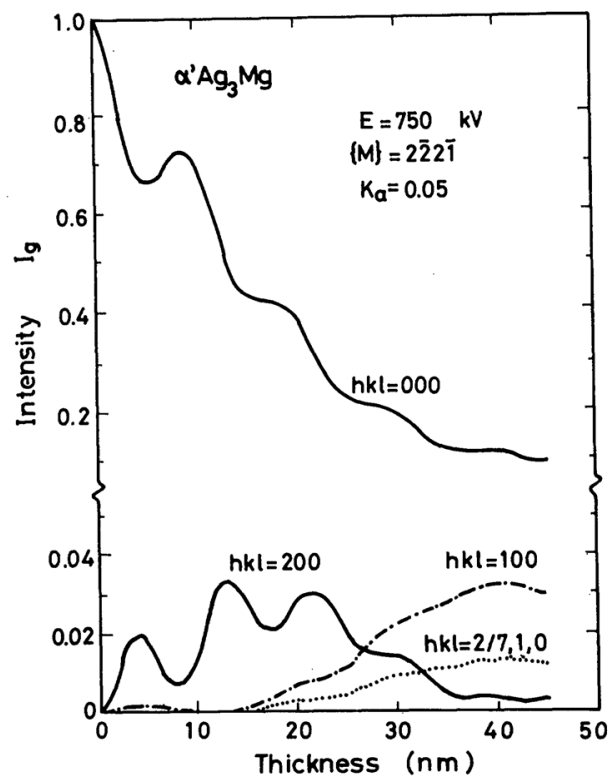

Fig. 1 Change in intensities of electron beams with thickness. Incident beam along [001]. fundamental reflections at about $T=40 \mathrm{~nm}$. Similar results have been obtained for $\alpha^{\prime \prime}$ $\mathrm{Cu}_{3} \mathrm{Pd}, \alpha_{2} \mathrm{Cu}_{3} \mathrm{Al}$ by us, and for $\mathrm{Au}-\mathrm{Cd}^{(13)}$. In order to obtain lattice images which reflect the superstructure in the sample, there must be a sizable amount of contribution to the image formation by the superlattice reflections. Therefore, for the observation of domain structures under an electron microscope a fairly thicker sample is necessary than the one used in the ordinary lattice image formation.

Figure 2 shows an example of the computer simulation for lattice images of $\alpha^{\prime} \mathrm{Ag}_{3} \mathrm{Mg}$ of various thicknesses. In the case of a thin sample, the lattice sites of fundamental $f c c$ lattice lose distinct contrast because of the instability in accelerating voltage or in objective lense current, which is expressed by

Thickness

$8.2 \mathrm{~nm}$

$16.5 \mathrm{~nm}$

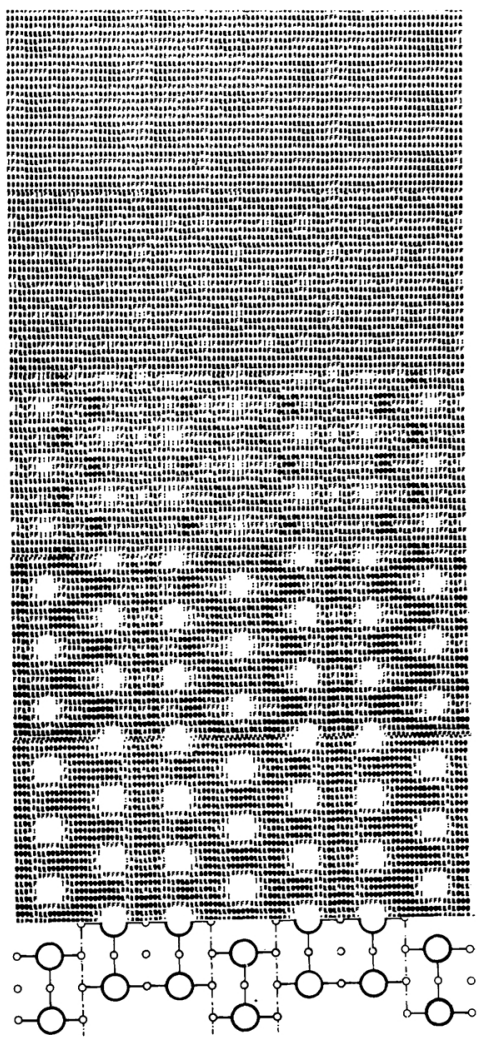

$24.7 \mathrm{~nm}$

$32.9 \mathrm{~nm}$

$41.2 \mathrm{~nm}$

$$
\mathrm{OMg} \circ \mathrm{Ag}
$$

Fig. 2 Computer simulation of the lattice images of $\alpha^{\prime} \mathrm{Ag}-\mathrm{Mg}$. Change with the crystal thickness. $E=$ $750 \mathrm{kV}, C_{s}=10 \mathrm{~mm}, f_{\Delta}=120 \mathrm{~nm}, \Delta=20 \mathrm{~nm}, q_{0}=$ 0 , aperture radius $=5 \mathrm{~nm}^{-1}$. 
$\Delta$ in eq. (4). In the image for thicker samples with $T$ of about $40 \mathrm{~nm}$ dotted contrast appears which corresponds to $\mathrm{Mg}$ sites, as clarified by comparison of the images with the model shown at the bottom of the figure. It must be emphasized here that in the lattice images of superstructural alloys the lattice sites of minority atoms appear with contrast. The situation could be understood qualitatively by the following kinematical consideration. The structure factor $F(\boldsymbol{h})$ of a binary substitutional alloy $\mathrm{A}-\mathrm{B}$, with atomic scattering factors $f_{a}$ and $f_{b}$, may be defined and rewritten in the following way:

$$
\begin{aligned}
\boldsymbol{F}(\boldsymbol{h})= & \sum_{\boldsymbol{j}} f_{j} \exp \left(2 \pi i \boldsymbol{h} \cdot \boldsymbol{r}_{\boldsymbol{j}}\right) \\
= & \sum_{j} \bar{f} \exp \left(2 \pi i \boldsymbol{h} \cdot \boldsymbol{r}_{\boldsymbol{j}}\right) \\
& +\sum_{j_{\boldsymbol{a}}} C_{b}\left(f_{a}-f_{b}\right) \exp \left(2 \pi i \boldsymbol{h} \cdot \boldsymbol{r}_{\boldsymbol{j}_{a}}\right) \\
& -\sum_{\boldsymbol{j}_{\boldsymbol{b}}} C_{\boldsymbol{a}}\left(f_{\boldsymbol{a}}-f_{b}\right) \exp \left(2 \pi i \boldsymbol{h} \cdot \boldsymbol{r}_{j_{b}}\right),
\end{aligned}
$$

where $C_{a}$ and $C_{b}$ are the atomic fractions of $\mathrm{A}$ and $\mathrm{B}$ atoms, respectively, and $\bar{f}$ is the mean atomic scattering factor defined by $\bar{f}=C_{a} f_{a}+$ $C_{b} f_{b}$. In the expression (6) the first term is the structure factor for fundamental reflection, and the second and third compose the one for superlattice reflection. Because the lattice images of superstructure are chiefly determined by interference between fundamental reflections including the transmitted wave and superlattice reflections, signs and absolute magnitudes of fictitious scattering factors appearing in the second and third terms in the expression (6) are important. They have opposite signs, and if $C_{a}>C_{b}$ the one at the atomic site $j_{b}$ for B atoms is larger in magnitude than the one at $j_{a}$ for A atoms. Thus the former appear with contrast in the lattice images, whereas the latter contribute to the contrast in the opposite sense. This explains why the minority members of component atoms, namely $\mathrm{Mg}$ atoms in Fig. 2, claim their positions as dots in the images.

Examples of actual lattice images of $\alpha^{\prime}$ $\mathrm{Ag}-\mathrm{Mg}$ are shown in Figs. 3(a) and 3(b). The distance between the columns of white dots is $0.4 \mathrm{~nm}$ and confirms that the dots correspond to $\mathrm{Mg}$ sites. Measured values of $\bar{M}$ are 1.75 for 3(a) and 1.83 for 3(b), but both of the images show the structures of a mixture
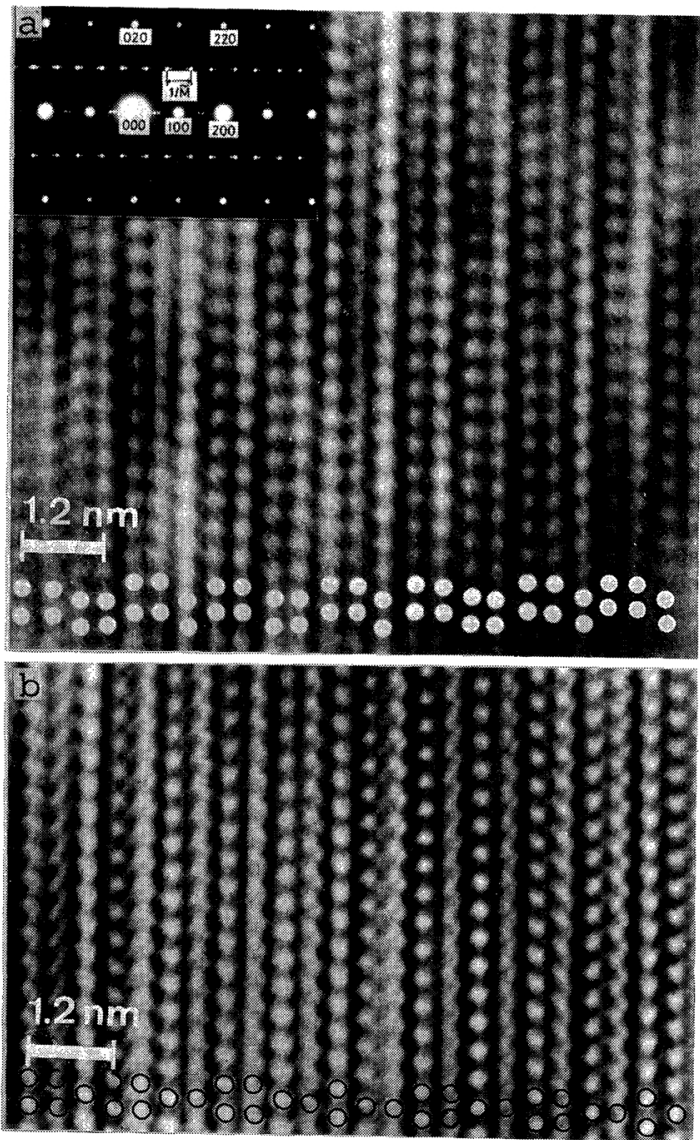

Fig. 3 Observed images of $\alpha^{\prime} \mathrm{Ag}-\mathrm{Mg}$. (a) Beam along [001]. $E=1000 \mathrm{kV}$. (b) Tilted sample. $E=750 \mathrm{kV}$.

of domains of $M=2$ and $M=1$. Portier et al. ${ }^{(14)(15)}$ recently made similar observations. We should note, however, some distortion of contrast appearing in the image of Fig. 3(b); namely the image of each $\mathrm{Mg}$ atomic site, which should be crystallographically equivalent, does not necessarily appear with the same contrast. In Fig. 3(b) the dots on the right side of APB are more distinct than the ones on the left side. This effect can be explained as due to a slight inclination of the sample, as is demonstrated by the following computer simulation.

Figure 4 shows the intensities of diffracted electron beams, when the sample is placed under the Bragg condition $h k l=100$, or when the incident beam is slanted by $\lambda / 2 a=0.0013$ $\operatorname{rad}$ from $z$ to $x$ axis of the crystal. As seen in the figure, the intensities of the diffracted beams with the same $h$ but of opposite signs are largely 


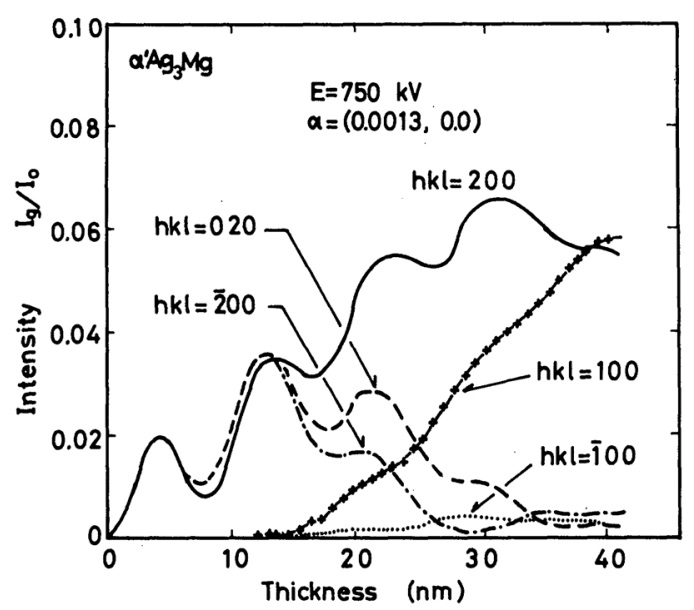

Fig. 4 Effect of tilting on intensities of electron beams. The sample is tilted so that the Bragg condition for 100 reflection is satisfied.

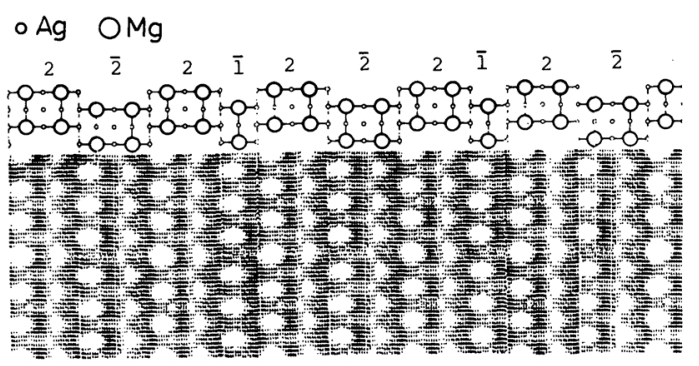

Fig. 5 Simulated lattice image of tilted sample. $f_{\Delta}=120 \mathrm{~nm}, \Delta=20 \mathrm{~nm}, q_{0}=0.1 \mathrm{~nm}^{-1}$.

different in magnitude, when the thickness of the sample is more than $20 \mathrm{~nm}$. An example of computer simulation of lattice images of slightly tilted sample of thickness $40 \mathrm{~nm}$ is shown in Fig. 5, which demonstrates the distortion of image contrast.

As observed frequently in $\alpha_{2} \mathrm{Cu}-\mathrm{Al}^{(16)}$, $\mathrm{Au}-\mathrm{Zn}^{(17)}$ and $\mathrm{Ag}-\mathrm{Mg}^{(14)}$, APB's often appear with jogs, and as a result APB's are not always straight lines in the images. The present authors examined ${ }^{(18)}$ the image contrast when one APB jogs and another APB penetrates obliquely the sample as illustrated in Fig. 6(a). We assumed that the crystal consists of several blocks as shown in Fig. 6(b). Each block is composed of (1)-type structure from the top to the depth $T_{f}$ and (2)-type from $T_{f}$ to the bottom, and $T_{f}$ varies stepwise from one block to another. The computed image under the
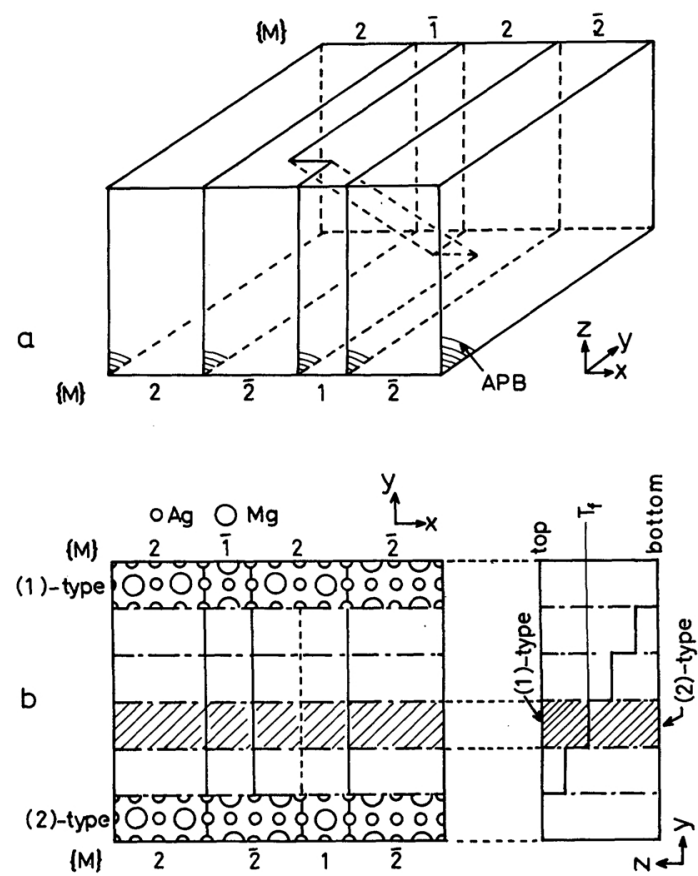

Fig. 6 Structure model for jogs of APB.

above conditions is shown in Fig. 7. One should note that the bright dots for $\mathrm{Mg}$ sites gradually lose their contrast as the step comes close to the middle depth of the sample. It must be emphasized, therefore, that the disappearance of dots at certain $\mathrm{Mg}$ sites does not necessarily mean depletion of atoms nor local disorder of constituent atoms.

\section{Antiphase Domain Structure in Ordered Alloys}

Many authors including the present ones have observed antiphase domain structures in various ordered alloys. For example, Mihama ${ }^{(19)}$, Watanabe and Takashima ${ }^{(20)}$, and Guymont et al. ${ }^{(21)}$ observed APB's with frequent jogs in CuAuII. In the alloys such as $\mathrm{Au}-\mathrm{Zn}^{(17)(22)}, \alpha^{\prime} \mathrm{Ag}-\mathrm{Mg}^{(14)(23)}, \alpha^{\prime \prime} \mathrm{Cu}-\mathrm{Pd}^{(24)}$, $\mathrm{Cu}-\mathrm{Sn}^{(25)}$, and $\alpha_{2} \mathrm{Cu}-\mathrm{Al}^{(16)(26)}$, the long period superstructures of nonintegral $\bar{M}$ values are made from a mixture of domains of local sizes with two adjacent integer $M$ values, and their APB's are not always perfect planes, but sometimes look curved because of successive jogs. Figure 8 shows an interesting example of 


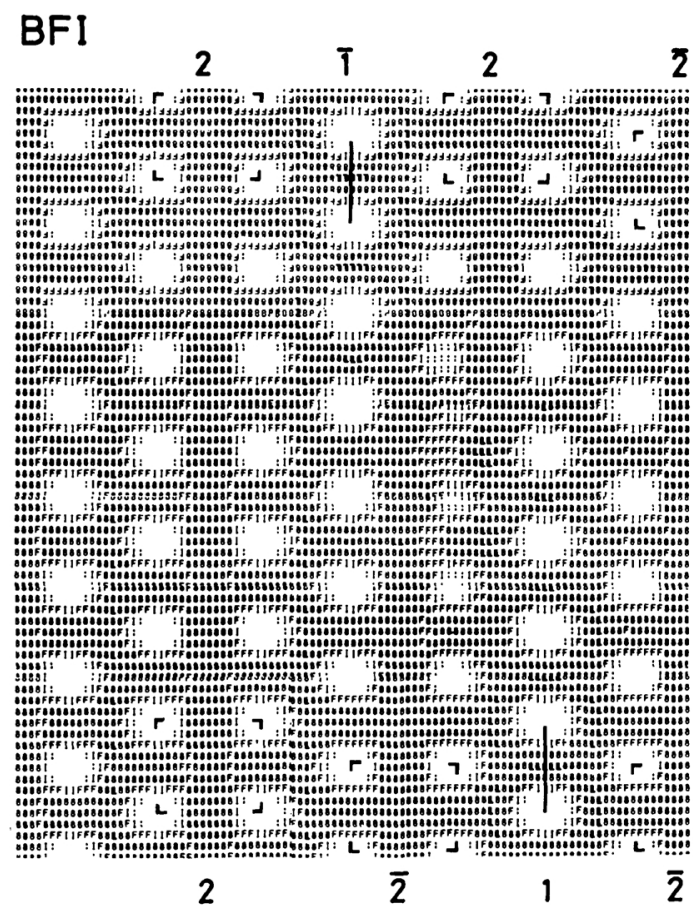

Fig. 7 Simulated lattice image of the structure shown in Fig. 6(b). $f_{\Delta}=120 \mathrm{~nm}, \Delta=20 \mathrm{~nm}, q_{0}=1 \mathrm{~nm}^{-1}$.

domain structure we have found in $\alpha_{2} \mathrm{Cu}-\mathrm{Al}$. The measured $\bar{M}$ value for this sample is 4.3 , which means that the structure is composed of the domains with $M=4$ and $M=5$. In the figure a set of eight domains of $M=5$, which look like lines with black contrast, unite together to disappear in the matrix of domains of $M=4$. The interpretation for the phenomenon has been given elsewhere ${ }^{(16)(26)}$. We have also an example of the case in which ten $M=4$ domains are united in the $M=5$ matrix $^{(16)}$.

In the region of the sample where APB's do not jog the antiphase structure is composed of domains lined up parallel in one direction. In such cases the structure is most conveniently described by the sequence of $M$ values, denoted as $\{M\}$ hereafter. In Table 1 a few examples of the domain structures expressed by $\{M\}$ in various alloys are given. From the table it can be understood that in the alloys with nonintegral $\bar{M}$ values, only those domains exist with local size $M_{1}=[\bar{M}]$ and $M_{2}=[\bar{M}]+1$, where $[\bar{M}]$ means the integer part of $\bar{M}$.

Once the fact is accepted, another way to

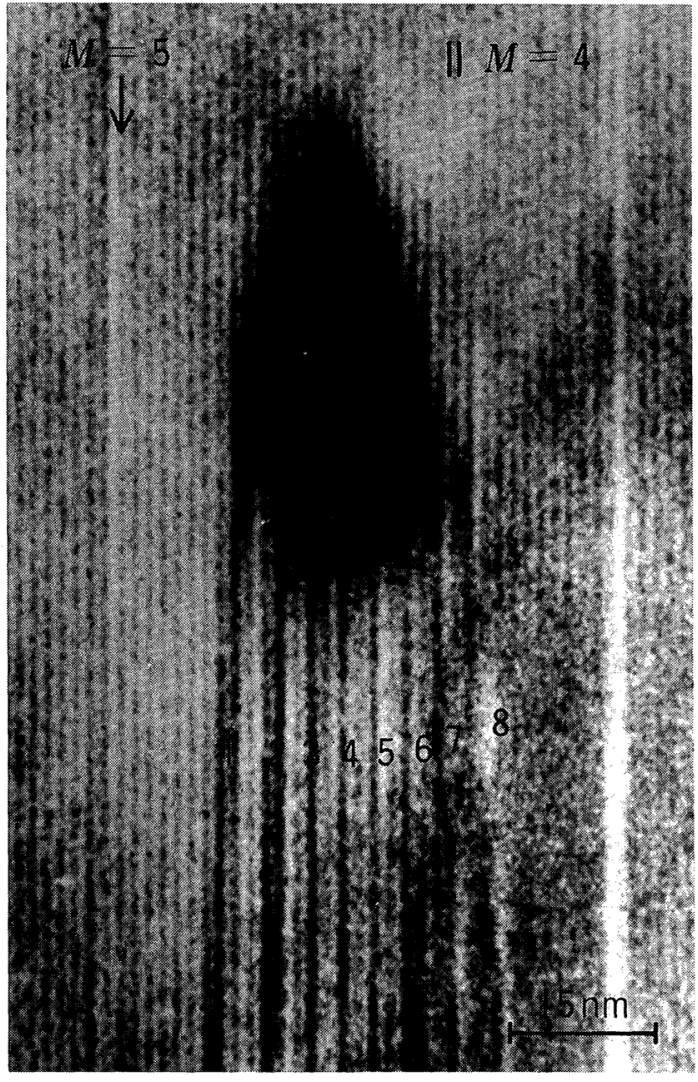

Fig. 8 Antiphase domain structure of $\alpha_{2} \mathrm{Cu}-\mathrm{Al}$. $E=500 \mathrm{kV}$.

Table 1 Examples of the arrangement of domains in $\alpha_{2} \mathrm{Cu}-\mathrm{Al}, \alpha^{\prime} \mathrm{Ag}-\mathrm{Mg}$ and $\alpha^{\prime \prime} \mathrm{Cu}-\mathrm{Pd}$.

$$
\begin{aligned}
& \alpha_{2} \mathrm{Cu}-\mathrm{Al} \quad(\bar{M}=4.36) \\
& \{M\}=\begin{array}{l}
545445445454545454545454454454 \\
454544445454454444445 \underbrace{444545454}_{n=3} \\
5445445-\ldots-
\end{array} \\
& \{n\}=122111111122214126311122 \ldots \\
& \alpha^{\prime} \mathrm{Ag}-\mathrm{Mg} \quad(\bar{M}=1.76) \\
& \{M\}=\begin{array}{l}
122212222122212222122212221222 \\
122212221222122212221222122212 \\
2212221-\ldots
\end{array} \\
& \{n\}=3434333333333333 \cdots-\cdots \\
& \alpha \text { 'Cu-Pd }(\bar{M}=3.69) \\
& \begin{array}{l}
\{M\}=\begin{array}{l}
443434434434443443443444344344 \\
344434344344434434443443443443 \\
4443443---
\end{array} \\
\{n\}=212232232231232322232-\ldots
\end{array}
\end{aligned}
$$


describe the sequence of domain sizes is to count the numbers $n$ of the majority members between two neighboring minority domains, and to express the sequence of $n$-values, or $\{n\}$, as shown in Table 1. From the table for $\{n\}$ we can easily conclude that the way of mixture of $M_{1}$ - and $M_{2}$-domains is uniform and almost, but not quite, regular in the sense defined by Fujiwara ${ }^{(27)}$.

\section{Distribution of Domain Sizes}

It is of interest to examine the distribution of domain sizes $M_{1}$ and $M_{2}$ in the long period superstructure whose average half period is $\bar{M}$. If the distribution be uniform and regular, then the sequence $\{n\}$ would be regular or periodic, as could be seen by further iterations. In reality, however, the distribution of $n$ values is neither regular nor periodic, and often consists of more than two components, showing the irregularity in the sequence $\{M\}$.

If, on the other hand, we assume a random distribution of $M_{1}$ - and $M_{2}$-domains, the distribution function for $n$-values would be given by a Poisson type, or

$$
P(n)=\frac{1}{n !}\left(\frac{1-x}{x}\right)^{n} \exp \left(-\frac{1-x}{x}\right),
$$

where $x$ is the fractional number of minority domiains, as given by the smaller one of either $\bar{M}-[\bar{M}]$ or $[\bar{M}]+1-\bar{M}$. Comparison of the actually observed distribution for $n$ with the Poisson distribution (7) reveals that the former is much sharper than the latter, and besides that in the actual cases two minority members never come in direct neighbors. This fact seems to indicate a repulsive force acting between two minority domains as suggested by $\mathrm{Kittel}^{(28)}$.

Thus we assume such a strong and shortranged repulsive interaction between neighboring minority domains, that they never come closer than a definite distance, or, in terms of $n$-value, $n$ should never be smaller than a definite integer $r$, and outside the "range" $r$ no interaction is at all assumed. Then the distribution function for $n$ is given by the following expression:

$$
P_{r}(n)=\left\{\begin{array}{l}
0 \text { for } n<r \\
\frac{1}{(n-r) !}\left(\frac{1-x-r x}{x}\right)^{n-r} \exp \left(-\frac{1-x-r x}{x}\right) \quad \text { for } n \geqq r .
\end{array}\right.
$$

We should note that for $r=0$ the expression (8) coincides with (7), and that both expressions naturally satisfy the following conditions:

$$
\begin{aligned}
& P(n) \equiv P_{0}(n), \\
& \sum_{n} P_{r}(n)=1,
\end{aligned}
$$

and

$$
\bar{n}=\sum_{n} n P_{r}(n)=\frac{1-x}{x} .
$$

In Fig. 9 some of our results on the observed distribution $f(n)$ of $n$-values are shown together with the ones predicted by $P(n)$ and $P_{r}(n)$. From the comparison made so far we see that $P_{r}(n)$ with $r=[\bar{n}]=[(1-x) / x]$ reproduces the observed distribution fairly satisfactorily.

\section{Conclusion}

The lattice images of the alloys with one- dimensional long period superstructures and their computer simulation suggest the following implications:

(1) In order to obtain good lattice images of the ordered structures, the samples must be rather thick.

(2) In the lattice images of superstructures atomic sites of the minority component usually show up with contrast.

(3) A slight tilting of the specimen results in distortion of the dotted contrast for atomic sites.

(4) Intersection of jogs of APB sometimes causes change in contrast at certain atomic positions.

(5) Alloys with nonintegral $\bar{M}$ values have domain structures of a mixture of two kinds of domains whose local sizes are either $[\bar{M}]$ or $[\bar{M}]+1$.

(6) The way of the above mixture can be conveniently expressed by the sequence $\{n\}$, 


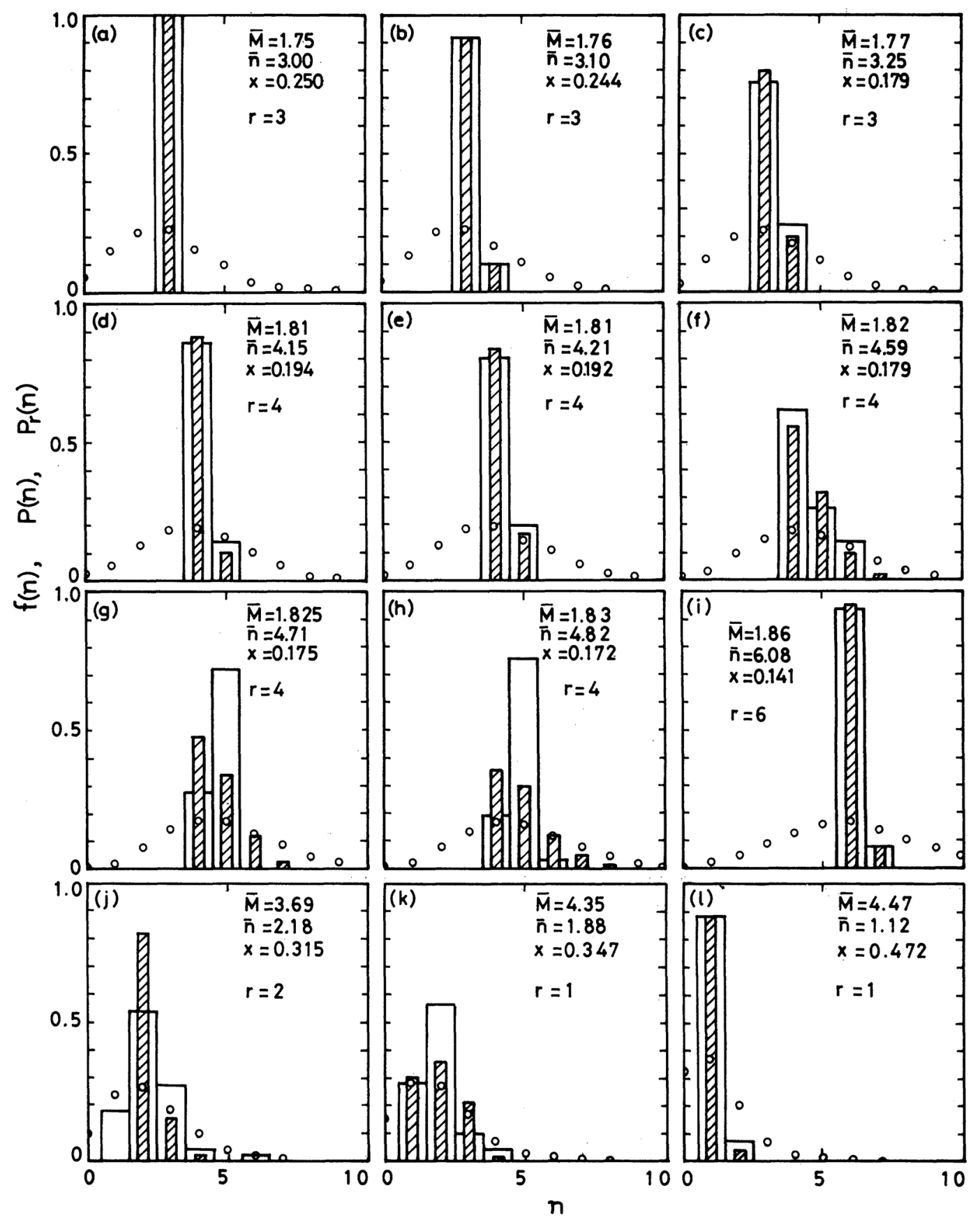

Fig. 9 Distributions of $n$ in $\alpha^{\prime} \mathrm{Ag}-\mathrm{Mg}(\mathrm{a}-\mathrm{i})$ and $\alpha^{\prime \prime} \mathrm{Cu}-\mathrm{Pd}(\mathrm{j}-l) . f(n)$ : observed result, $\Pi ; P(n)$ : Poisson distribution, $\bigcirc ; P_{r}(n)$ : distribution calculated from eq. (8), 츠:

where $n$ is the number of the majority members between two neighboring minority domains.

(7) The distribution of $n$-values indicates that the domain structures are uniform and almost, but not quite, regular, and that two minority domains never come to direct neighbors of each other.

(8) A trial theoretical distribution function 
is suggested to describe the observed distribution of $n$-values.

\section{Acknowledgments}

Thanks are due to all staff members and technicians belonging to the Research Laboratory of High Voltage Electron Microscope at Kyushu University for their assistance and cooperation rendered to us in performing the present investigation.

\section{REFERENCES}

(1) H. Sato and R. S. Toth: Long Period Superstructures in Alloys; Alloying Behavior and Effects in Concentrated Solid Solutions, ed. T. B. Masalski, Gordon and Breach Science Pub. Inc., New York, (1965), p. 380.

(2) E. Bernard and P. Duval: Phys. Status Solidi, (a) 34 (1976), 135

(3) N. Kuwano, T. Doi and T. Eguchi: Trans. JIM, 18 (1977), 807.

(4) H. Sato and R. S. Toth: Phys. Rev., 124 (1964), 1833.

(5) S. Ogawa, D. Watanabe, H. Watanabe and T. Komoda: Acta Cryst., 11 (1958), 872.

(6) D. W. Pashley and A. E. B. Presland: J. Inst. Metals, 87 (1958-59), 419.

(7) J. M. Cowley and A. F. Moodie: Acta Cryst., 10 (1957), 609.

(8) J. M. Cowley: Diffraction Physics, North Holland Pub. Co., Amsterdam, (1975), p. 221.

(9) P. A. Doyle and P. S. Turner: Acta Cryst., A 24 (1968), 390.

(10) P. L. Fejes: Acta Cryst., A 33 (1977), 109.

(11) K. Ishizuka: Ultramicroscopy, 5 (1980), 55.
(12) O. Scherzer: J. Appl. Phys., 20 (1949), 20.

(13) K. Hiraga, D. Shindo and M. Hirabayashi: J. Appl. Cryst., 14 (1981), 185.

(14) R. Portier, D. Gratias, M. Guymont and W. M. Stobbs: Acta Cryst., A 36 (1980), 190.

(15) R. Portier, D. Gratias, M. Guymont and W. M. Stobbs: J. Microscopy, 119 (1980), 163.

(16) N. Kuwano, H. Mishio, M. Toki and T. Eguchi: Phys. Satus Solidi, (a) 65 (1981), 341.

(17) G. van Tendeloo and S. Amelinckx: Phys. Status Solidi, (a) 50 (1978), 53.

(18) N. Kuwano, S. Sasaki, Y. Tomokiyo and T. Eguchi: Proc. 10th International Congress on Electron Microscopy, Hamburg 1982, (1982), p. 43.

(19) K. Mihama: J. Phys. Soc. Japan, 31 (1971), 1677.

(20) D. Watanabe and K. Takashima: J. Appl. Cryst., 8 (1975), 598.

(21) M. Guymont, R. Portier and D. Gratias: Acta Cryst., A 36 (1980), 792.

(22) G. van Tendeloo and S. Amelinckx: Phys. Status Solidi, (a) 43 (1977), 553.

(23) M. Guymont, R. Portier, D. Gratias and W. M. Stobbs: Modulated Structures 1979, AIP Conf. Proc., No. 53 (1979), p. 244 \& 256.

(24) T. Eguchi, K. Oki, Y. Tomokiyo and N. Kuwano: Sci. Rep. Res. Inst. Tohoku Univ., A 29, Suppl. 1 (1980), 25.

(25) M. van Sande, R. de Ridder, G. van Tendeloo, J. van Landuyt and S. Amelinckx: Phys. Status Solidi, (a) 48 (1978), 383.

(26) N. Kuwano, H. Mishio and T. Eguchi: Modulated Structures 1979, AIP Conf. Proc., No. 53 (1979), p. 273.

(27) K. Fujiwara: J. Phys. Soc. Japan, 12 (1957), 7.

(28) C. Kittel: Solid State Commun., 25 (1978), 519 \& 31 (1979), 121. 